\title{
Beobachtungen zur Frage der morphologischen und funktionellen Asymmetrie des menschlichen Körpers.
}

Von

\author{
Emil Abderhalden, Halle a. S. \\ (Eingegangen am 10. Juni 1919.)
}

In einer interessanten Abhandlung hat F. O. Guldberg ${ }^{1}$ ) eine ganze Reihe von Beobachtungen über Zirkularbewegungen von Menschen und Tieren gesammelt. Menschen und Tiere, die aus irgendeinem Grunde der Orientierung mittels ihrer Sinnesorgane beraubt sind, sei es, dass die Umgebung als solche keine Anhaltspunkte gibt, sei es, dass Dunkelheit, Schneestürme, Nebel usw. den Gebrauch des Gesichtssinnes unmöglich machen, kehren im Bogen zur Ausgangsstelle zurück. Als Ursache der Kreisbewegung wird die morphologische und funktionelle Asymmetrie des Körpers angeführt. Guld berg fasst die Kreisbewegung als eine-der wichtigsten Bedingungen für die Erhaltung des Lebens auf. Die jungen Tiere treffen wieder auf die Alten; sofern diese in der Nähe der Ausgangsstelle des Wanderns sich aufhalten.

Kürzlich ist Pintner ${ }^{2}$ ) der Ursache nachgegangen, die dem Umstande zugrunde lag, dass die Verordnung in Wien, links zu gehen, fortwährend durchbrochen wurde. Immer wieder wurde trotz aller Verordnungen und aller Ermahnungen die rechte Gehrichtung gewählt. Während das Rechtsgehen und Rechtsausweichen ohne besondere Willensanstrengung sich ganz von selbst einstellt, brauchen die weitaus meisten Menschen, um links zu gehen, eines besonderen, und zwar starken Willensimpulses. Auch hier spielt zweifellos die Asymmetrie des Körpers in morphologischer und funktioneller Hinsicht die ausschlaggebende Rolle.

Ich konnte in den letzten Jahren eine grosse Zahl von Beobachtungen ausführen, die im gleichen Sinne liegen. Zum physiologischen Institute der Universität Halle führen zwei breite, bequeme, steinerne Treppen. Die Treppenanlage ist genau symmetrisch gebaut. Die eine Treppe führt rechts, die andere links herauf. Es war nun von Interesse,

1) F. O. Guld berg, Die Zirkularbewegung als tierische Grundbewegung, ihre Ursache, Phänomenalität und Bedeutung. Zeitschr. f. Biologie Bd. 35 S. 419. 1897.

2) Pintner, Vorträge des Vereins zur Verbreitung naturwissenschaftl. Kenntnisse in Wien, Jahrg. 58, Heft 1. 1918, nach einem Referat in der Naturwissenschaftl. Wochenschr. Bd. 34 S. 226. 1919. 
festzustellen, wie oft die rechte und wie oft die linke Treppe benutzt wird. Zu diesem Zwecke zählte ich in den einzelnen Semestern - besonders in den ersten Tagen - die Zahl der Studenten, die die rechte oder linke Treppe emporstiegen. Die Ergebnisse waren ganz eklatant. Die bei weitem grösste Zahl der.Studierenden benutzte regelmässig die rechte Treppe. Nur ganz wenige erklommen die linke. Am eindeutigsten war das Resultat, solange Einzelpersonen ankamen. Wurde der Andrang stärker, dann benutzten manche die linke Treppe, um rascher emporzukommen. Interessant war, dass die Linkshänder mit wenigen Ausnahmen die linke Treppe benutzten.

Einige Beobachtungen mögen das Mitgeteilte belegen:

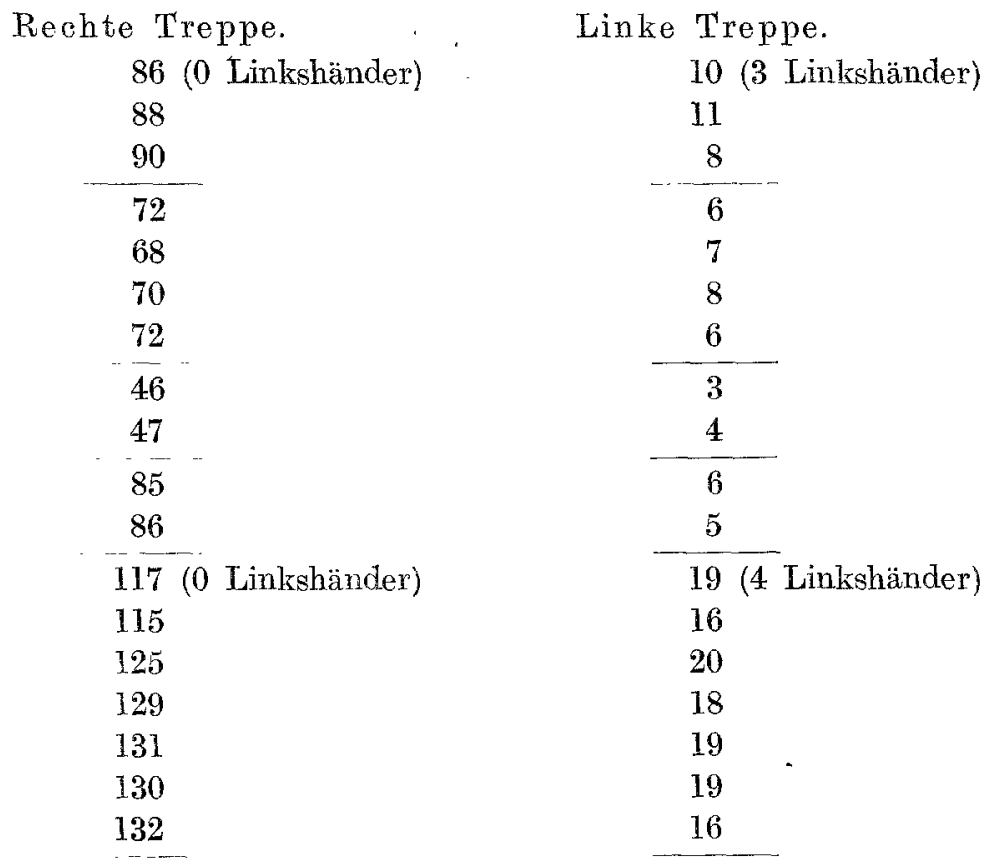

Noch grösser werden die Unterschiede, wenn diejenigen Perioden fortgelassen werden, während deren ein grosser Andrang stattfand, und. infolgedessen mancher die linke Treppe wählte, nưr um rascher emporzukommen.

So benutzten zum Beispiel, gezählt vom ersten Ankömmling an, 41. Studierende die rechte Treppe und. nur einer (ein Linkshänder) die linke. Dann folgte ein grosser Andrang, wobei die Treppe in Gruppen erstiegen wurde. 55 Personen kamen die rechte Treppe herauf und 16 die linke. Dann liess der Andrang nach. In der Folgezeit benutzten 43 Personen die rechte und nur 2 die linke Treppe. Ein Unterschied 
im Verhalten der beiden Geschlechter war nicht feststellbar. Es kamen zum Beispiel 11 Studentinnen rechts und 2 links herauf.

Es sei noch ausdrücklich betont, dass nicht etwa die eine" oder andere Treppe in der Richtung der Ankommenden liegt. Vielmehr stehen die Treppen im rechten Winkel zu einer kleinen, beiden gemeinsamen Anfangstreppe.

In sehr grossem Umfange konnten auch Beobachtungen an einem gemischten Publikum gemacht werden. Im physiologischen Institut ist die Geschäftsstelle des Bundes zur Erhaltung und Mehrung der Volks kraft untergebracht. Sie wird zum Zweck der Ackerpachtung oder der Abholung von Sämereien usw. in gewissen Zeiten von Hunderten von Personen besucht. Ferner fanden wiederholt Sitzungen und Vorträge im physiologischen Institute statt. Es seien einige dieser Beobachtungen mitgeteilt :

Rechte Treppe.

568

227

890

212

145 (darunter 1 Linkshänder). 98

714

58

64

168 (darunter 0 Linkshänder).

171

415
Linke Treppe.

- 65

33

72

26

27 (darunter 8 Linkshänder). 12

23

2

1

24. (darunter 7 Linkshänder). 12

26

Die Feststellung, ob linkshändig oder rechtshändig, liess sich leider nur selten durchführen. Es mussten die Leute beim Heraufkommen beobachtet und gezählt werden. Gleichzeitig mussten für die auf der linken Seite ankommenden Personen bestimmte Merkmale der Kleidung oder des Aussehens vermerkt werden. Dann wurde festgestellt, ob sie mit der rechten oder linken Hand sich in Listen eintrugen. Die einfache Aufforderung, zu melden, ob links- oder rechtshändig, hatte kein eindeutiges Resultat.

Interessant ist nun die Feststellung, dass beim Verlassen des Institutes der Unterschied in der Benutzung der rechten oder linken Treppe fast ganz fortfällt. So benutzten von 146 Personen 72 die rechte und 74 die linke Treppe. Auch dann, wenn in grossen Abständen. Einzelpersonen die Treppe benutzten, wurde keine Seite ausgesprochen bevorzugt. Es stellte sich allmählich eine Gewöhnung ein. Bestimmte Personen gingen immer rechts, andere immer links herunter. Das 
Emporsteigen erfordert mehr Anstrengung als dos Herabsteigen. Es ist wohl möglich, dass dieser Umstand das verschiedene Verhalten bestimmt.

Interessant war mir auch, dass Maueranschläge immer rechts und nie links vom Treppenaufgang angebracht wurden. Die Personen, die sie anbrachten, rechneten offenbar damit, dass die meisten Personen die rechte Treppe benutzen.

Schon vor mehreren Jahren (1899) machte ich Versuche mit Linksund Rechtshändern, deren Augen verdeckt waren, im freien Gelände, um festzustellen, ob der zurückgelegte Bogen in beiden Füllen verschieden, d. h. das eine Mal rechts- und das andere Mal linksherum geschlossen wurde. Die Ergebnisse waren nicht eindeutig, weil die Geländeflächen zu klein waren. Die Versuchspersonen liefen an Hindernisse und orientierten sich dann. Immerhin schien ein Unterschied vorhanden zu sein. Es müsste nicht schwer sein, dieser Frage beim Rudern auf einem grossen See bei verbundenen Augen nachzugehen (wobei allerdings nur die Arme in Frage kämen) oder auf einer grossen. Sand- oder Eisfläche.

Interessant wäre es auch, an einem grossen Material zu prüfen, welches Bein beim Tasten benutzt wird. Geht man im Dunkeln an einem unbekannten Orte, dann prüft man häufig mit den Beinen, ob ein Hindernis droht. Es ist dies besonders dann der Fall, wenn in einem Gang eine Treppe zu erwarten ist. Ich persönlich verwende das rechte Bein. Sobald man sein Augenmerk ausdrücklich auf die Verwendung der Beine zu Tastzwecken wendet, dann ist die unvoreingenommene Beobachtung gestört. Aus diesem Grunde ist es schwer, ausreichende Beobachtungen zu sammeln. 